Journal of Mathematics and Statistics 8 (1): 92-97, 2012

ISSN 1549-3644

(C) 2012 Science Publications

\title{
Multiparameterized Soft Set
}

\author{
Abdul Razak Salleh, \\ Shawkat Alkhazaleh, Nasruddin Hassan and Abd Ghafur Ahmad \\ School of Mathematical Sciences, Faculty of Science and Technology \\ University Kebangsaan Malaysia, 43600 UKM Bangi, Selangor DE, Malaysia
}

\begin{abstract}
Problem statement: In 1999 Molodtsov introduced the concept of a soft set as a general mathematical tool for dealing with uncertainty. The solutions of such problems involve the use of mathematical principles based on uncertainty and imprecision. Approach: In this study we recall the definition of a soft set, its properties and its operations. Results: As a generalization of Molodtsov's soft set we introduce the definition of a multiparameterized soft set and its basic operations, namely complement, union, intersection and OR. Conclusion: We give examples for these concepts. Basic properties of the operations are also given.
\end{abstract}

Key words: Soft set, multisoft set, basic operations, basic properties

\section{INTRODUCTION}

Most of the problems in engineering, medical science, economics, environments. have various uncertainties. Molodtsov (1999) initiated the concept of soft set theory as a mathematical tool for dealing with uncertainties which is free from the above difficulties. After Molodtsov's work, some different operations and application of soft sets were studied by Chen et al. (2005), Maji et al. (2002, 2003). Furthermore Maji et al. (2001) presented the definition of fuzzy soft set and Roy and Maji (2007) presented the applications of this notion to decision making problems. As a generalization of Molodtsov's soft set we introduce the definition of a multisoft set and its basic operations, namely complement, union and intersection. We give examples for these concepts. Basic properties of the operations are also given.

Preliminaries: We recall some basic notions in soft set theory. Molodtsov (1999) defined soft set in the following way. Let $\mathrm{U}$ be a universe set and let $\mathrm{E}$ be a set of parameters. Let $\mathrm{P}(\mathrm{U})$ denote the power set of $\mathrm{U}$ and $\mathrm{A} \subseteq \mathrm{E}$.

Definition 1: (Molodtsov, 1999). A pair (F, A) is called a soft set over $U$, where $F$ is a mapping given by $F$ : $\mathrm{A} \rightarrow \mathrm{P}(\mathrm{U})$. In other words, a soft set over $\mathrm{U}$ is a parameterized family of subsets of the universe $U$ for $\varepsilon \in \mathrm{A}, \mathrm{F}(\varepsilon)$ may be considered as the set of $\varepsilon$ approximate elements of the soft set $(\mathrm{F}, \mathrm{A})$.
Example 1: Let us consider a soft set (F, E) which describes the "attractiveness of houses"' that Mr. $\mathrm{X}$ is considering for purchase. Suppose that there are six houses in the universe $\mathrm{U}=\left\{\mathrm{H}_{1}, \mathrm{H}_{2}, \mathrm{H}_{3}, \mathrm{H} 4, \mathrm{H}_{5}, \mathrm{H}_{6}\right\}$ under consideration and that $\mathrm{E}=\left\{\mathrm{e}_{1}, \mathrm{e}_{2}, \mathrm{e}_{3}, \mathrm{e}_{4}, \mathrm{e}_{5}\right\}$ is a set of decision parameters. The $e_{i}(1,2,3,4,5)$ stand for the parameters "expensive', 'beautiful', "wooden", "cheap" and "in green surroundings", respectively.

Consider the mapping F given by "houses (.)', where (.) is to be filled in by one of the parameters $E_{i} \in E$, For instance, $F\left(e_{1}\right)$ means "houses (expensive)" and its functional value is the set $\{h \in U: h$ is an expensive house

Suppose that $\mathrm{F}(\mathrm{e} 1)=\left\{\mathrm{h}_{2}, \mathrm{~h}_{4}\right\}, \mathrm{F}\left(\mathrm{e}_{2}\right)=\left\{\mathrm{h}_{1}, \mathrm{~h}_{3}\right\}, \mathrm{F}\left(\mathrm{e}_{3}\right)=$ $\varnothing, \mathrm{F}\left(\mathrm{e}_{4}\right)=\left\{\mathrm{h}_{1}, \mathrm{~h}_{3}, \mathrm{~h}_{5}\right\}$ and $\mathrm{F}\left(\mathrm{e}_{5}\right)=\left\{\mathrm{H}_{1}\right\}$. Then we can view the soft set $(\mathrm{F}, \mathrm{E})$ as consisting of the following collection of approximations:

$$
\begin{aligned}
(\mathrm{F}, \mathrm{E})=\{ & \left(\text { expensive houses, }\left\{\mathrm{h}_{2}, \mathrm{~h}_{4}\right\}\right), \\
& \left(\text { beautiful houses, }\left\{\mathrm{h}_{1}, \mathrm{~h}_{3}\right\}\right), \\
& (\text { wooden houses, } \varnothing), \\
& \left(\text { cheap houses, }\left\{\mathrm{h}_{1}, \mathrm{~h}_{3}, \mathrm{~h}_{5}\right\}\right), \\
& \left.\left(\text { in the green surroundings, }\left\{\mathrm{h}_{1}\right\}\right)\right\} .
\end{aligned}
$$

Each approximation has two parts: a predicate and an approximate value set.

The following definitions are due to Maji et al. (2003).

Corresponding Author: Abdul Razak Salleh, School of Mathematical Sciences, Faculty of Science and Technology, University Kebangsaan, Malaysia Tel: 03-8921 5757 Fax :03-8925 4519 
Definition 2: For two soft sets $(\mathrm{F}, \mathrm{A})$ and $(\mathrm{G}, \mathrm{B})$ over $\mathrm{U},(\mathrm{F}, \mathrm{A})$ is called a soft subset of $(\mathrm{G}, \mathrm{B})$ if:

- $\mathrm{A} \subset \mathrm{B}$ and

- $\forall \varepsilon \in \mathrm{A}, \mathrm{F}(\varepsilon)$ and $\mathrm{G}(\varepsilon)$ are identical approximations

This relationship is denoted by $(F, A) \tilde{\subset}(G, B)$. In this case $(\mathrm{G}, \mathrm{B})$ is called a soft superset of $(\mathrm{F}, \mathrm{A})$ and is denoted by $(\mathrm{G}, \mathrm{B}) \tilde{\supset}(\mathrm{F}, \mathrm{A})$.

Definition 3: Two soft sets ( $F, A)$ and $(G, B)$ over a common universe $U$ are said to be soft equal if $(F, A)$ is a soft subset of $(G, B)$ and $(G, B)$ is a soft subset of $(F, A)$.

Definition 4: Let $E=\left\{e_{1}, e_{2}, \ldots, e_{n}\right\}$ be a set of parameters. The NOT set of $\mathrm{E}$, denoted by ${ }_{7} \mathrm{E}$, is defined

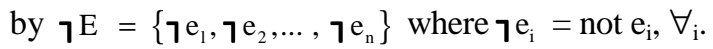

Definition 5: The complement of a soft set $(\mathrm{F}, \mathrm{A})$ is denoted by $(\mathrm{F}, \mathrm{A})^{\mathrm{c}}$ and is defined by $\left.(\mathrm{F}, \mathrm{A})^{\mathrm{c}}=\left(\mathrm{F}^{\mathrm{c}},\right\rceil^{\mathrm{A}}\right)$ where $\mathrm{F}^{\mathrm{c}}: \mathrm{\eta}_{\mathrm{A}} \rightarrow \mathrm{P}(\mathrm{U})$ is a mapping given by $\left.\left.\mathrm{F}^{\mathrm{c}}(\alpha)=\mathrm{U}-\mathrm{F}(\rceil^{\alpha}\right), \forall \alpha \in\right\rceil^{\mathrm{A}} \mathrm{A}$.

Definition 6: A soft set (F, A) over $U$ is said to be a null soft set, denoted by $\Phi$, if $\forall \varepsilon \in A, F(\varepsilon)=\varnothing$ ( null-set).

Definition 7: A soft set (F, A) over $U$ is said to be an absolute soft set, denoted by $\tilde{A}$, if $\forall \varepsilon \in A, F(\varepsilon)=U$.

Definition 8: The union of two soft sets $(F, A)$ and $(G$, B) over a common universe $U$ is the soft set $(H, C)$ where $\mathrm{C}=\mathrm{A} \cup \mathrm{B}$, and $\forall \varepsilon \in \mathrm{C}$ :

$$
H(\varepsilon)=\left\{\begin{array}{lcc}
F(\varepsilon) & \text { if } \quad \varepsilon \in A-B \\
G(\varepsilon) & \text { if } \quad \varepsilon \in B-A \\
F(\varepsilon) \cup G(\varepsilon) & \text { if } \quad \varepsilon \in A \cap B
\end{array}\right.
$$

Definition 9: (Ali et al., 2009). The extended intersection of two soft sets $(F, A)$ and $(G, B)$ over a common universe $\mathrm{U}$ is the soft set $(\mathrm{H}, \mathrm{C})$ where $\mathrm{C}=\mathrm{A} \cup \mathrm{B}$, and $\forall \varepsilon \in \mathrm{C}$ :

$$
H(\varepsilon)=\left\{\begin{array}{lcc}
F(\varepsilon) & \text { if } & \varepsilon \in A-B \\
G(\varepsilon) & \text { if } & \varepsilon \in B-A \\
F(\varepsilon) \cap G(\varepsilon) & \text { if } & \varepsilon \in A \cap B
\end{array}\right.
$$

Note: The definition of intersection of two soft sets given by Maji et al. (2003) was not correct because they defined $\mathrm{H}(\varepsilon)=\mathrm{F}(\varepsilon)$ or $\mathrm{G}(\varepsilon)$. This was pointed out by Ali et al. (2009). In fact also gave the definition of restricted intersection.

Definition 10: If $(F, A)$ and $(G, B)$, are two soft sets, then $(F, A)$ and $(G, B)$, denoted by $(F, A) \wedge(G, B)$, is defined by:

$$
(\mathrm{F}, \mathrm{A}) \wedge(\mathrm{G}, \mathrm{B})=(\mathrm{H}, \mathrm{A} \times \mathrm{B})
$$

where $\mathrm{H}(\alpha, \beta)=\mathrm{F}(\alpha) \cap \mathrm{G}(\beta) \forall(\alpha, \beta) \in \mathrm{A} \times \mathrm{B}$.

Definition 11: If $(\mathrm{F}, \mathrm{A})$ and $(\mathrm{G}, \mathrm{B})$ are two soft sets, then $(\mathrm{F}, \mathrm{A}) \mathrm{OR}(\mathrm{G}, \mathrm{B})$ denoted by $(\mathrm{F}, \mathrm{A}) \wedge(\mathrm{G}, \mathrm{B})$, is defined by:

$$
(\mathrm{F}, \mathrm{A}) \wedge(\mathrm{G}, \mathrm{B})=(\mathrm{H}, \mathrm{A} \times \mathrm{B})
$$

where $H(\alpha, \beta)=F(\alpha) \cup G(\beta) \forall(\alpha, \beta) \in A \times B$.

Multiparameterized soft set: We introduce the definition of a multiparameterized soft set and its basic operations, namely complement, union, intersection and and OR. We give examples for these concepts. Basic properties of the operations are derived.

Let $U$ be a universe set and $E_{i}$ be a set of parameters for $i \in I$ such that $\bigcap_{i \in I} E_{i}=\varnothing$. Let $P(U)$ denote the power set of $\mathrm{U}, \mathrm{E}=\bigcup_{\mathrm{i} \in \mathrm{I}} \mathrm{E}_{\mathrm{i}}, \mathrm{P}(\mathrm{E})$ denotes the power set of $\mathrm{E}$ and $\mathrm{A} \subset \mathrm{P}(\mathrm{E})$.

Definition 12: A pair ( $F, A$ ) is called a multiparameterized soft set over $\mathrm{U}$ where $\mathrm{F}$ is a mapping given by $\mathrm{F}: \mathrm{A} \rightarrow \mathrm{P}(\mathrm{U})$. In other words, a multiparameterized soft set over $U$ is a parameterized family of subsets of the universe $U$ for $\varepsilon \in A, F(\varepsilon)$ may be considered as the set of $\varepsilon$-approximate sets of the multiparameterized soft set $(\mathrm{F}, \mathrm{A})$.

Example 2: Let us consider a multiparameterized soft set $(F, E)$ which describes the " attractiveness of houses in the state of Selangor, Malaysia' that Mr. $\mathrm{X}$ is considering for purchase. Suppose that there are ten houses in the universe:

$$
\mathrm{U}=\left\{\mathrm{h}_{1}, \mathrm{~h}_{2}, \mathrm{~h}_{3}, \mathrm{~h}_{4}, \mathrm{~h}_{5}, \mathrm{~h}_{6}, \mathrm{~h}_{7}, \mathrm{~h}_{8}, \mathrm{~h}_{9}, \mathrm{~h}_{10}\right\}
$$

Under consideration and that $E_{i}=\left\{E_{1}, E_{2}, E_{3}\right\}$ is a set of decision parameters. Let $\mathrm{E}_{1}$ be a set of cost parameters given by $\mathrm{E}_{1}=\left\{\mathrm{e}_{1,1}=\right.$ expensive, $\mathrm{e}_{1,2}=$ 
cheap, $e_{1,3}$ very expensive, $e_{1,4}=$ very cheap $\} E_{2}$ is a set of location parameters given by $\mathrm{E}_{2}=\left\{\mathrm{e}_{2,1}=\right.$ Subang Jaya, $\mathrm{e}_{2,2}=$ Klang, $\mathrm{e}_{2,3}=$ Ampang, $\mathrm{e}_{2,4}=$ Shah Alam, $\mathrm{e}_{2,5}$ $=$ Kajang $\}$ and $E_{3}$ is a set of color parameters given by $E_{3}=\left\{e_{3,1}=\right.$ green, $e_{3,2}=$ dark green, $e_{3,3}=$ blue, $e_{3,5}=$ red $\}$. Let $E=\bigcup_{i} E_{i}$ and $A \subseteq P(E)$ such that:

$$
\begin{gathered}
\mathrm{A}=\left\{\mathrm{a}_{1}=\left\{\mathrm{e}_{1,1}\right\}, \mathrm{a}_{2}=\left\{\mathrm{e}_{1,1}, \mathrm{e}_{2,1}\right\}, \mathrm{a}_{3}=\left\{\mathrm{e}_{1,1}, \mathrm{e}_{2,1}, \mathrm{e}_{3,1}\right\},\right. \\
\mathrm{a}_{4}=\left\{\mathrm{e}_{1,2}\right\}, \mathrm{a}_{5}=\left\{\mathrm{e}_{1,2}, \mathrm{e}_{2,1}, \mathrm{e}_{3,3}\right\}, \\
\left.\mathrm{a}_{6}=\left\{\mathrm{e}_{1,4}, \mathrm{e}_{2,4}\right\}, \mathrm{a}_{7}=\left\{\mathrm{e}_{1,2}, \mathrm{e}_{3,2}\right\}\right\} .
\end{gathered}
$$

Suppose that $\mathrm{F}\left(\alpha_{1}\right)=\left\{\mathrm{h}_{2}, \mathrm{~h}_{3}, \mathrm{~h}_{6}, \mathrm{~h}_{7}, \mathrm{~h}_{10}\right\}, \mathrm{F}\left(\alpha_{2}\right)=$ $\left\{\mathrm{h}_{6}, \mathrm{~h}_{7}\right\}, \mathrm{F}\left(\alpha_{3}\right)=\varnothing \mathrm{F}\left(\alpha_{4}\right)=\left\{\mathrm{h}_{1}, \mathrm{~h}_{8}, \mathrm{~h}_{9}\right\}, \mathrm{F}\left(\alpha_{5}\right)=\left\{\mathrm{h}_{8}\right\}$, $F\left(\alpha_{6}\right)=\varnothing$ and $F\left(\alpha_{7}\right)=\left\{h_{9}\right\}$ Then we can view the multiparameterized soft set $(\mathrm{F}, \mathrm{A})$ as consisting of the following collection of approximations:

$$
\begin{aligned}
&(\mathrm{F}, \mathrm{A})=\left\{\left(\mathrm{a}_{1},\left\{\mathrm{~h}_{2}, \mathrm{~h}_{3}, \mathrm{~h}_{6}, \mathrm{~h}_{7}, \mathrm{~h}_{10}\right\}\right),\left(\mathrm{a}_{2},\left\{\mathrm{~h}_{6}, \mathrm{~h}_{7}\right\}\right),\right. \\
&\left(\mathrm{a}_{3}, \varnothing\right),\left(\mathrm{a}_{4},\left\{\mathrm{~h}_{1}, \mathrm{~h}_{8}, \mathrm{~h}_{9}\right\}\right), \\
&\left.\left(\mathrm{a}_{5},\left\{\mathrm{~h}_{8}\right\}\right),\left(\mathrm{a}_{6}, \varnothing\right),\left(\mathrm{a}_{7},\left\{\mathrm{~h}_{9}\right\}\right)\right\} .
\end{aligned}
$$

Each approximation has two parts: a predicate and an approximate value set.

Definition 13: For two multiparameterized soft sets (F, A) and $(\mathrm{G}, \mathrm{B})$ over $\mathrm{U},(\mathrm{F}, \mathrm{A})$ is called a multiparameterized soft subset of $(G, B)$ if:

- $\mathrm{A} \subset \mathrm{B}$ and

- $\forall \varepsilon \subset A, \quad F \quad(\varepsilon)$ and $G \quad(\varepsilon)$ are identical approximations

This relationship is denoted by $(\mathrm{F}, \mathrm{A}) \tilde{\subset}(\mathrm{G}, \mathrm{B})$. In this case $(G, B)$ is called a multiparameterized soft superset of $(\mathrm{F}, \mathrm{A})$ denoted by $(\mathrm{G}, \mathrm{B}) \tilde{\mathrm{D}}(\mathrm{F}, \mathrm{A})$.

Definition 13: Two multiparameterized soft sets (F, A) and $(\mathrm{G}, \mathrm{B})$ over a common universe $\mathrm{U}$ are said to be multiparameterized soft equal if $(\mathrm{F}, \mathrm{A})$ is a multiparameterized soft subset of $(\mathrm{G}, \mathrm{B})$ and $(\mathrm{G}, \mathrm{B})$ is a multiparameterized soft subset of $(\mathrm{F}, \mathrm{A})$.

Example 3: Let $\mathrm{A}=\left\{\alpha_{1}, \alpha_{4}, \alpha_{7}\right\}$ and $\mathrm{B}=\left\{\alpha_{1}, \alpha_{2}, \alpha_{3}\right.$, $\left.\alpha_{4}, \alpha_{5}, \alpha_{7}\right\} \subset \mathrm{P}(\mathrm{E})$ clearly, $\mathrm{A} \subset \mathrm{B}$. Let (F, A) and (G, B) be two multiparameterized soft sets over the same universe $\mathrm{U}=\left\{\mathrm{h}_{1}, \mathrm{~h}_{2}, \mathrm{~h}_{3}, \mathrm{~h}_{4}, \mathrm{~h}_{5}, \mathrm{~h}_{6}, \mathrm{~h}_{7}, \mathrm{~h}_{8}, \mathrm{~h}_{9}, \mathrm{~h}_{10},\right\}$ such that $(\mathrm{F}, \mathrm{A})=\left\{\left(\alpha_{1},\left\{\mathrm{~h}_{2}, \mathrm{~h}_{6}, \mathrm{~h}_{10}\right\}\right),\left(\alpha_{4}\left\{\mathrm{~h}_{1}, \mathrm{~h}_{8}\right\}\right),\left(\alpha_{7}\right.\right.$, $\left.\left.\left\{\mathrm{h}_{9}\right\}\right)\right\}$ and:

$$
\begin{aligned}
(\mathrm{G}, \mathrm{B})=\left\{\left(\mathrm{a}_{1},\left\{\mathrm{~h}_{2}, \mathrm{~h}_{3}, \mathrm{~h}_{6}, \mathrm{~h}_{7}, \mathrm{~h}_{10}\right\}\right),\left(\mathrm{a}_{2},\left\{\mathrm{~h}_{6}, \mathrm{~h}_{7}\right\}\right),\right. \\
\\
\left(\mathrm{a}_{3}, \varnothing\right),\left(\mathrm{a}_{4},\left\{\mathrm{~h}_{1}, \mathrm{~h}_{8}, \mathrm{~h}_{9}\right\}\right),\left(\mathrm{a}_{5},\left\{\mathrm{~h}_{8}\right\}\right), \\
\left.\left(\mathrm{a}_{6}, \varnothing\right),\left(\mathrm{a}_{7},\left\{\mathrm{~h}_{9}\right\}\right)\right\}
\end{aligned}
$$

Therefore $(\mathrm{F}, \mathrm{A}) \tilde{\subset}(\mathrm{G}, \mathrm{B})$

Definition 14: Let $P(E)$ be a power set of param- eters. The not set of $\mathrm{P}(\mathrm{E})$ denoted by ${ }_{7} \mathrm{P}(\mathrm{E})$, is defined by

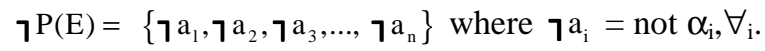

Example 4: Consider the example as presented in Example 2. The:

$$
\begin{aligned}
& { }_{\mathbf{7}} \mathrm{A}=\left\{\boldsymbol{7}^{\mathrm{a}_{1}}=\left\{\boldsymbol{7} \mathrm{e}_{1,1}\right\}, \boldsymbol{7} \mathrm{a}_{2}=\left\{\boldsymbol{7}_{\mathrm{1}, 1}, \boldsymbol{7} \mathrm{e}_{2,1}\right\},\right. \\
& \boldsymbol{\eta}^{\mathrm{a}_{3}}=\left\{\boldsymbol{\eta}_{\mathrm{i}, 1}, \boldsymbol{\eta}_{\mathrm{e}, 1}, \mathrm{e}_{3,1}\right\}, \\
& \mathrm{\eta}^{\mathrm{a}} \mathrm{u}_{4}=\left\{\mathrm{\eta}_{\mathrm{1}, 2}\right\}, \\
& \mathbf{7}_{5}=\left\{\boldsymbol{\eta}_{1,2}, \boldsymbol{\eta} \mathrm{e}_{2,1}, \boldsymbol{\eta} \mathrm{e}_{3,3}\right\} \text {, } \\
& \boldsymbol{\eta}_{6}=\left\{\boldsymbol{\eta}_{1,4}, \boldsymbol{\eta} \mathrm{e}_{2,4}\right\} \text {, } \\
& \left.\boldsymbol{\eta}^{\mathrm{a}}=\left\{\boldsymbol{\eta}_{1,2}, \boldsymbol{\eta} \mathrm{e}_{3,2}\right\}\right\} \text {. }
\end{aligned}
$$

Definition 15: The complement of amultiparameterized soft set $(\mathrm{F}, \mathrm{A})$ is denoted by $(\mathrm{F}, \mathrm{A})^{\mathrm{c}}$ and is defined by $(\mathrm{F}, \mathrm{A})^{\mathrm{c}}=\left(\mathrm{F}^{\mathrm{c}},{ }_{7} \mathrm{~A}\right)$ where $\left.\mathrm{F}^{\mathrm{c}}:\right\rceil \mathrm{A} \rightarrow \mathrm{P}(\mathrm{U})$ is mapping given by $\mathrm{F}^{\mathrm{c}}(\alpha)=\mathrm{U}-\mathrm{F}\left(\boldsymbol{\eta}^{\alpha}\right), \forall \alpha \subset \boldsymbol{\eta}^{\mathrm{A}}$.

Example 5: Consider the example as presented in Example 2. Then we have:

$$
\begin{aligned}
(F, A)^{c}=\{ & \left(7 a_{1},\left\{F\left(7 a_{1}\right)\right\}\right),\left(7 a_{2},\left\{F\left(7 a_{2}\right)\right\}\right), \\
& \left(7 a_{3},\left\{F\left(7 a_{3}\right)\right\}\right),\left(7 a_{4},\left\{F\left(7 a_{4}\right)\right\}\right), \\
& \left(7 a_{5},\left\{F\left(7 a_{5}\right)\right\}\right),\left(7 a_{6},\left\{F\left(7 a_{6}\right)\right\}\right), \\
& \left.\left(7 a_{7},\left\{F\left(7 a_{7}\right)\right\}\right)\right\} .
\end{aligned}
$$

Definition 16: A multiparameterized soft set (F, A) over $U$ is said to be a nullmultipara- meterized soft set denoted by $\Phi$ if $\forall \varepsilon \in \mathrm{A}, \mathrm{F}(\varepsilon)=\varnothing$ (null-set).

Example 6: Suppose that there are ten houses in the universe $U$, where $U$ is the set of red and expensive houses under consideration in Kajang. Let $\mathrm{E}_{1}, \mathrm{E}_{2}, \mathrm{E}_{3}$ be sets of cost, locations and colours parameters respectively where $\mathrm{E}_{1}=\left\{\mathrm{e}_{1,1}=\right.$ Expensive, $\mathrm{e}_{1,2}=$ cheap, $\mathrm{e}_{1.3}=$ very expensive, $\mathrm{e}_{1.4}=$ very cheap $\}, \mathrm{E}_{2}=\left\{\mathrm{e}_{2,1}=\right.$ Subang Jaya, $\mathrm{e}_{2,2}=$ Klang, $\mathrm{e}_{2,3}=$ Ampang, $\mathrm{e}_{2,4}=$ Shah 
Alam, $\mathrm{e}_{2,5}=$ Kajang $\} \mathrm{E}_{3}=\left\{\mathrm{e}_{3,1}=\right.$ Green, $\mathrm{e}_{3,2}=$ dark green, $e_{3,3}=$ blue, $e_{3,4}=$ light blue. Let $E=\bigcup_{i} E_{i}$ and $A$ $\subseteq \mathrm{P}(\mathrm{E})$ such that:

$$
\begin{aligned}
\mathrm{A}=\left\{\mathrm{a}_{1}\right. & =\left\{\mathrm{e}_{3,1}\right\}, \mathrm{a}_{2}=\left\{\mathrm{e}_{1,1}, \mathrm{e}_{2,1}, \mathrm{e}_{3,3}\right\}, \\
\mathrm{a}_{3} & =\left\{\mathrm{e}_{1,1}, \mathrm{e}_{2,1}, \mathrm{e}_{3,1}\right\}, \mathrm{a}_{4}=\left\{\mathrm{e}_{1,2}, \mathrm{e}_{3,2}\right\}, \\
\mathrm{a}_{5} & =\left\{\mathrm{e}_{1,2}, \mathrm{e}_{2,1}, \mathrm{e}_{3,3}\right\}, \mathrm{a}_{6}=\left\{\mathrm{e}_{1,4}, \mathrm{e}_{3,1}\right\}, \\
\mathrm{a}_{7} & \left.=\left\{\mathrm{e}_{1,2}, \mathrm{e}_{3,2}\right\}\right\} .
\end{aligned}
$$

The multiparameterized soft set $(\mathrm{F}, \mathrm{A})$ is the collection of approximations as given below:

$$
F\left(\alpha_{1}\right)=\varnothing, F\left(\alpha_{2}\right)=\varnothing, \ldots, F\left(\alpha_{7}\right)=\varnothing
$$

Thus $(\mathrm{F}, \mathrm{A})$ is a null multiparameterized soft set.

Definition 17: A multiparameterized soft set (F, A) over $\mathrm{U}$ is said to be an absolute multipar- ameterized soft set denoted by $\tilde{\mathrm{A}}$, if $\forall \varepsilon \in \mathrm{A}, \mathrm{F}(\varepsilon)=\mathrm{U}$.

Example 7: Suppose that there are ten houses in the universe $U$ where $U$ is the set of red and expensive houses under consideration in Kajang. Let $E_{1}$ be a set of cost parameters such that $\mathrm{E}_{1}=\left\{\mathrm{e}_{1,1}=\right.$ expensive, $\mathrm{e}_{1,2}=$ cheap, $e_{1,3}$ very expensive, $e_{1,4}=$ very cheap $\} E_{2}$ is a set of location parameters such that $\mathrm{E}_{2}=\left\{\mathrm{e}_{2,1}=\right.$ Subang Jaya, $\mathrm{e}_{2,2}=$ Klang, $\mathrm{e}_{2,3}=$ Ampang, $\mathrm{e}_{2,4}=$ Shah Alam, $\mathrm{e}_{2,5}$ $=$ Kajang $\}$ and $E_{3}$ is a set of colour parameters such that $\mathrm{E}_{3}=\left\{\mathrm{e}_{3,1}=\right.$ green, $\mathrm{e}_{3,2}=$ dark green, $\mathrm{e}_{3,3}=$ blue, $\mathrm{e}_{3,4}$ light blue, $e_{3,5}=$ red $\}$ Let $E=\bigcup_{i} E_{i}$ and $A \subseteq P(E)$, such that:

$$
\begin{aligned}
\mathrm{A}=\left\{\mathrm{a}_{1}\right. & =\left\{\mathrm{e}_{1,1}\right\}, \mathrm{a}_{2}=\left\{\mathrm{e}_{1,1}, \mathrm{e}_{2,5}\right\}, \\
\mathrm{a}_{3} & =\left\{\mathrm{e}_{1,1}, \mathrm{e}_{2,5}, \mathrm{e}_{3,5}\right\}, \\
\mathrm{a}_{4} & =\left\{\mathrm{e}_{2,5}, \mathrm{e}_{3,5}\right\}, \mathrm{a}_{5}=\left\{\mathrm{e}_{1,1}, \mathrm{e}_{3,5}\right\}, \\
\mathrm{a}_{6} & \left.=\left\{\mathrm{e}_{3,1}\right\}, \mathrm{a}_{7}=\left\{\mathrm{e}_{2,5}\right\}\right\} .
\end{aligned}
$$

The multiparameterized soft set $(\mathrm{F}, \mathrm{A})$ is the collection of approximations as given below:

$$
\mathrm{F}\left(\mathrm{a}_{1}\right)=\mathrm{U}, \mathrm{F}\left(\mathrm{a}_{2}\right)=\mathrm{U}, \ldots, \mathrm{F}\left(\mathrm{a}_{7}\right)=\mathrm{U}
$$

Then $(\mathrm{F}, \mathrm{A})$ is an absolute multiparameterized soft set.

Definition 18: The union of two multiparamet- erized soft sets $(F, A)$ and $(G, B)$ over a common universe $U$ is the multiparameterized soft set $(\mathrm{H}, \mathrm{C})$ where $\mathrm{C}=\mathrm{A} \cup \mathrm{B}$, and $\forall \varepsilon \in \mathrm{C}$ :

$$
H(\varepsilon)=\left\{\begin{array}{llc}
F(\varepsilon) & \text { if } & \varepsilon \in A-B, \\
G(\varepsilon) & \text { if } & \varepsilon \in \mathrm{B}-\mathrm{A}, \\
F(\varepsilon) \cup \mathrm{G}(\varepsilon) & \text { if } & \varepsilon \in \mathrm{A} \cap \mathrm{B} .
\end{array}\right.
$$

Example 8: Consider the example as presented in Example 3.1. Let (F, A) and $(\mathrm{G}, \mathrm{B})$ be two multiparameterized soft sets over the same universe $\mathrm{U}$ $=\left\{\mathrm{h}_{1}, \mathrm{~h}_{2}, \mathrm{~h}_{3}, \mathrm{~h}_{4}, \mathrm{~h}_{5}, \mathrm{~h}_{6}, \mathrm{~h}_{7}, \mathrm{~h}_{8}, \mathrm{~h}_{9}, \mathrm{~h}_{10},\right\}$ such that:

$$
\begin{gathered}
\mathrm{A}=\left\{\mathrm{a}_{1}=\left\{\mathrm{e}_{1,1}\right\}, \mathrm{a}_{2}=\left\{\mathrm{e}_{1,1}, \mathrm{e}_{2,1}\right\},\right. \\
\mathrm{a}_{3}=\left\{\mathrm{e}_{1,1}, \mathrm{e}_{2,1}, \mathrm{e}_{3,1}\right\}, \mathrm{a}_{4}=\left\{\mathrm{e}_{1,2}\right\}, \\
\mathrm{a}_{5}=\left\{\mathrm{e}_{1,2}, \mathrm{e}_{2,1}, \mathrm{e}_{3,3}\right\}, \mathrm{a}_{6}=\left\{\mathrm{e}_{1,4}, \mathrm{e}_{2,4}\right\}, \\
\left.\mathrm{a}_{7}=\left\{\mathrm{e}_{1,2}, \mathrm{e}_{3,2}\right\}\right\}, \\
\mathrm{B}=\left\{\mathrm{b}_{1}=\left\{\mathrm{e}_{1,1}, \mathrm{e}_{2,1}\right\}, \mathrm{b}_{2}=\left\{\mathrm{e}_{1,3}\right\}, \mathrm{b}_{3}=\left\{\mathrm{e}_{1,4}, \mathrm{e}_{2,4}\right\},\right. \\
\left.\mathrm{b}_{4}=\left\{\mathrm{e}_{1,1}, \mathrm{e}_{2,2}, \mathrm{e}_{3,3}\right\}, \mathrm{b}_{5}=\left\{\mathrm{e}_{1,2}, \mathrm{e}_{3,2}\right\}\right\} .
\end{gathered}
$$

Suppose that $\mathrm{F}\left(\alpha_{1}\right)=\mathrm{U}=\left\{\mathrm{h}_{1}, \mathrm{~h}_{3}, \mathrm{~h}_{6}, \mathrm{~h}_{7}, \mathrm{~h}_{10}\right\}, \mathrm{F}$ $\left(\alpha_{2}\right)=\left\{h_{6}, h_{7}\right\}, F\left(\alpha_{3}\right)=\varnothing, F\left(\alpha_{4}\right)=\left\{h_{1}, h_{8}, h_{9},\right\}, F\left(\alpha_{5}\right)$ $=\left\{h_{8}\right\}, F\left(\alpha_{6}\right)=\varnothing, F\left(\alpha_{7}\right)=\left\{h_{9}\right\}$ and $G\left(b_{1}\right)=\left\{h_{2}, h_{4}\right.$, $\left.\mathrm{h}_{6}, \mathrm{~h}_{8},\right\}, \mathrm{G}\left(\mathrm{b}_{2}\right)=\left\{\mathrm{h}_{10}\right\}, \mathrm{G}\left(\mathrm{b}_{3}\right)=\varnothing, \mathrm{G}\left(\mathrm{b}_{4}\right)=\left\{\mathrm{h}_{4}, \mathrm{~h}_{6}\right\}$ and $\mathrm{G}\left(\mathrm{b}_{5}\right)=\left\{\mathrm{h}_{8}, \mathrm{~h}_{9}\right\}$. Then:

$$
\begin{aligned}
\mathrm{C}=\mathrm{A} \cup \mathrm{B}=\left\{\mathrm{c}_{1}\right. & =\left\{\mathrm{e}_{1,1}\right\}, \mathrm{c}_{2}=\left\{\mathrm{e}_{1,1}, \mathrm{e}_{2,1}\right\}, \\
\mathrm{c}_{3} & =\left\{\mathrm{e}_{1,1}, \mathrm{e}_{2,1}, \mathrm{e}_{3,1}\right\}, \mathrm{c}_{4}=\left\{\mathrm{e}_{1,2}\right\}, \\
\mathrm{c}_{5} & =\left\{\mathrm{e}_{1,2}, \mathrm{e}_{2,1}, \mathrm{e}_{3,3}\right\}, \\
\mathrm{c}_{6} & =\left\{\mathrm{e}_{1,4}, \mathrm{e}_{2,4}\right\}, \mathrm{c}_{7}=\left\{\mathrm{e}_{1,2}, \mathrm{e}_{3,2}\right\}, \\
\mathrm{c}_{8} & \left.=\left\{\mathrm{e}_{1,3}\right\}, \mathrm{c}_{9}=\left\{\mathrm{e}_{1,1}, \mathrm{e}_{2,2}, \mathrm{e}_{3,3}\right\}\right\} .
\end{aligned}
$$

Hence the union of the two multiparameterized soft sets $(F, A)$ and $(G, B)$ over a common universe $\mathrm{U}$ is:

$$
\begin{aligned}
(\mathrm{H}, \mathrm{C})=\{ & \left(\mathrm{c}_{1},\left\{\mathrm{~h}_{2}, \mathrm{~h}_{3}, \mathrm{~h}_{6}, \mathrm{~h}_{7}, \mathrm{~h}_{10}\right\}\right), \\
& \left(\mathrm{c}_{2},\left\{\mathrm{~h}_{2}, \mathrm{~h}_{4}, \mathrm{~h}_{6}, \mathrm{~h}_{7}, \mathrm{~h}_{8}\right\}\right),\left(\mathrm{c}_{3}, \varnothing\right), \\
& \left(\mathrm{c}_{4},\left\{\mathrm{~h}_{1}, \mathrm{~h}_{8}, \mathrm{~h}_{9}\right\}\right), \\
& \left(\mathrm{c}_{5},\left\{\mathrm{~h}_{8}\right\}\right),\left(\mathrm{c}_{6}, \varnothing\right),\left(\mathrm{c}_{7},\left\{\mathrm{~h}_{8}, \mathrm{~h}_{9}\right\}\right), \\
& \left.\left(\mathrm{c}_{8},\left\{\mathrm{~h}_{10}\right\}\right),\left(\mathrm{c}_{9},\left\{\mathrm{~h}_{4}, \mathrm{~h}_{6}\right\}\right)\right\} .
\end{aligned}
$$

Definition 19: The intersection of two multiparameterized soft sets (F, A) and (G, B) over a common universe $\mathrm{U}$ is the soft set $(\mathrm{H}, \mathrm{C})$ where $\mathrm{C}=\mathrm{A} \cup \mathrm{B}$, and $\forall \varepsilon \in \mathrm{C}:$ 


$$
H(\varepsilon)= \begin{cases}F(\varepsilon) & \text { if } \quad \varepsilon \in A-B, \\ G(\varepsilon) & \text { if } \quad \varepsilon \in B-A, \\ F(\varepsilon) \cap G(\varepsilon) & \text { if } \quad \varepsilon \in A \cap B .\end{cases}
$$

Example 9: Let (F, A) and (G, B) be two multiparameterized soft sets over the same universe $U$ as given in Example 8. Then:

$$
\begin{aligned}
\mathrm{C}=\mathrm{A} \cup \mathrm{B}=\left\{\mathrm{c}_{1}\right. & =\left\{\mathrm{e}_{1,1}\right\}, \mathrm{c}_{2}=\left\{\mathrm{e}_{1,1}, \mathrm{e}_{2,1}\right\}, \\
\mathrm{c}_{3} & =\left\{\mathrm{e}_{1,1}, \mathrm{e}_{2,1}, \mathrm{e}_{3,1}\right\}, \mathrm{c}_{4}=\left\{\mathrm{e}_{1,2}\right\}, \\
\mathrm{c}_{5} & =\left\{\mathrm{e}_{1,2}, \mathrm{e}_{2,1}, \mathrm{e}_{3,3}\right\}, \\
\mathrm{c}_{6} & =\left\{\mathrm{e}_{1,4}, \mathrm{e}_{2,4}\right\}, \mathrm{c}_{7}=\left\{\mathrm{e}_{1,2}, \mathrm{e}_{3,2}\right\}, \\
\mathrm{c}_{8} & \left.=\left\{\mathrm{e}_{1,3}\right\}, \mathrm{c}_{9}=\left\{\mathrm{e}_{1,1}, \mathrm{e}_{2,2}, \mathrm{e}_{3,3}\right\}\right\} .
\end{aligned}
$$

Hence the intersection of $(F, A)$ and $(G, B)$ over $U$ is:

$$
\begin{aligned}
(\mathrm{O}, \mathrm{C})= & \left\{\left(\mathrm{c}_{1},\left\{\mathrm{~h}_{2}, \mathrm{~h}_{3}, \mathrm{~h}_{6}, \mathrm{~h}_{7}, \mathrm{~h}_{10}\right\}\right),\right. \\
& \left(\mathrm{c}_{2},\left\{\mathrm{~h}_{6}\right\}\right),\left(\mathrm{c}_{3}, \varnothing\right),\left(\mathrm{c}_{4},\left\{\mathrm{~h}_{1}, \mathrm{~h}_{8}, \mathrm{~h}_{9}\right\}\right), \\
& \left(\mathrm{c}_{5},\left\{\mathrm{~h}_{8}\right\}\right),\left(\mathrm{c}_{6}, \varnothing\right),\left(\mathrm{c}_{7},\left\{\mathrm{~h}_{9}\right\}\right), \\
& \left.\left(\mathrm{c}_{8},\left\{\mathrm{~h}_{10}\right\}\right),\left(\mathrm{c}_{9},\left\{\mathrm{~h}_{4}, \mathrm{~h}_{6}\right\}\right)\right\} .
\end{aligned}
$$

We now derive the following properties of union and intersection of multiparameterized soft sets.

Proposition 1: If $(\mathrm{F}, \mathrm{A}),(\mathrm{G}, \mathrm{B})$ and $(\mathrm{H}, \mathrm{C})$ are three multiparameterized soft sets over $U$ then:

- $\quad(\mathrm{F}, \mathrm{A}) \cup(\mathrm{G}, \mathrm{B})=(\mathrm{G}, \mathrm{B}) \cup(\mathrm{F}, \mathrm{A})$

- $(\mathrm{F}, \mathrm{A}) \cap(\mathrm{G}, \mathrm{B})=(\mathrm{G}, \mathrm{B}) \cap(\mathrm{F}, \mathrm{A})$

- $(\mathrm{F}, \mathrm{A}) \cup((\mathrm{G}, \mathrm{B}) \cup(\mathrm{H}, \mathrm{C}))=((\mathrm{F}, \mathrm{A}) \cup(\mathrm{G}, \mathrm{B})) \cup(\mathrm{H}, \mathrm{C})$

- $((\mathrm{F}, \mathrm{A}) \cap(\mathrm{G}, \mathrm{B})) \cap(\mathrm{H}, \mathrm{C})((\mathrm{F}, \mathrm{A}) \cap(\mathrm{G}, \mathrm{B})) \cap(\mathrm{H}, \mathrm{C})$

- $((\mathrm{F}, \mathrm{A}) \cup(\mathrm{G}, \mathrm{B})) \cap((\mathrm{F}, \mathrm{A}) \cup(\mathrm{H}, \mathrm{C}))$

$((\mathrm{F}, \mathrm{A}) \cup(\mathrm{G}, \mathrm{B})) \cap((\mathrm{F}, \mathrm{A}) \cup(\mathrm{H}, \mathrm{C}))$

- $\quad((\mathrm{F}, \mathrm{A}) \cap(\mathrm{G}, \mathrm{B})) \cup((\mathrm{F}, \mathrm{A}) \cap(\mathrm{H}, \mathrm{C}))$

$((\mathrm{F}, \mathrm{A}) \cap(\mathrm{G}, \mathrm{B})) \cup((\mathrm{F}, \mathrm{A}) \cap(\mathrm{H}, \mathrm{C}))$

Proof: Straightforward from definitions 18 and 19.

Definition 20: If (F, A) and (G, B) are two multiparameterized soft sets, then "(F, A) AND (G, B)" denoted by $(F, A) \wedge(G, B)$ is defined by, $(F, A) \wedge(G, B)$ $=(\mathrm{H}, \mathrm{A} \times \mathrm{B})$ where $\mathrm{H}(\alpha, \beta)=\mathrm{F}(\alpha) \cap \mathrm{G}(\beta) \forall(\alpha, \beta) \in \mathrm{A} \times \mathrm{B}$.

Example 10: Let us consider multiparameterized soft sets (F, A) and (G, B) which describes the "attractiveness of houses in the state of Selangor,
Malaysia" that Mr. X and Mr. Y considering for purchase respectively over the same universe $U=\left\{h_{1}\right.$, $\left.\mathrm{h}_{2}, \mathrm{~h}_{3}, \mathrm{~h}_{4}, \mathrm{~h}_{5}, \mathrm{~h}_{6}, \mathrm{~h}_{7}, \mathrm{~h}_{8}, \mathrm{~h}_{9}, \mathrm{~h}_{10},\right\}$ such that $\mathrm{A}=\left\{\alpha_{1}=\right.$ $\left\{\mathrm{e}_{1,1}\right\}, \alpha_{2}=\left\{\mathrm{e}_{1,1}, \mathrm{e}_{2,1}\right\}, \alpha_{3}=\left\{\mathrm{e}_{1,1}, \mathrm{e}_{2,1}, \mathrm{e}_{3,1}\right\} \quad \mathrm{B}=\left\{\mathrm{b}_{1}=\right.$ $\left.\left\{\mathrm{e}_{1,1}, \mathrm{e}_{2,1}\right\}, \mathrm{b}_{2}=\left\{\mathrm{e}_{1,3}\right\}, \mathrm{b}_{3}=\left\{\mathrm{e}_{1,4}, \mathrm{e}_{2,4}\right\}\right\}$.

Suppose that $F\left(\alpha_{1}\right)=\left\{h_{2}, h_{3}, h_{6}, h_{7}, h_{10}\right\}, F\left(\alpha_{2}\right)=$ $\left\{\mathrm{h}_{6}, \mathrm{~h}_{7}\right\}$ and $\mathrm{F}\left(\alpha_{3}\right)=\varnothing$ and $\mathrm{G}\left(\mathrm{b}_{1}\right)=\left\{\mathrm{h}_{2}, \mathrm{~h}_{4}, \mathrm{~h}_{6}, \mathrm{~h}_{8}\right\}, \mathrm{G}$ $\left(b_{2}\right)=\left\{h_{10}\right\}$ and $G\left(b_{3}\right)=\varnothing$, Then:

$$
\begin{aligned}
\mathrm{A} \times \mathrm{B}=\{ & \left\{\left(\mathrm{a}_{1}, \mathrm{~b}_{1}\right),\left(\mathrm{a}_{1}, \mathrm{~b}_{2}\right),\left(\mathrm{a}_{1}, \mathrm{~b}_{3}\right),\left(\mathrm{a}_{2}, \mathrm{~b}_{1}\right),\left(\mathrm{a}_{2}, \mathrm{~b}_{2}\right),\right. \\
& \left.\left(\mathrm{a}_{2}, \mathrm{~b}_{3}\right),\left(\mathrm{a}_{3}, \mathrm{~b}_{1}\right),\left(\mathrm{a}_{3}, \mathrm{~b}_{2}\right),\left(\mathrm{a}_{3}, \mathrm{~b}_{3}\right)\right\}
\end{aligned}
$$

Hence the $(\mathrm{F}, \mathrm{A}) \wedge(\mathrm{G}, \mathrm{B})$ over a common universe $\mathrm{U}$ is:

$$
\begin{aligned}
&(\mathrm{H}, \mathrm{A} \times \mathrm{B})=\left\{\left(\left(\mathrm{a}_{1}, \mathrm{~b}_{1}\right),\left\{\mathrm{h}_{2}, \mathrm{~h}_{6}\right\}\right),\left(\left(\mathrm{a}_{1}, \mathrm{~b}_{2}\right),\left\{\mathrm{h}_{10}\right\}\right),\right. \\
&\left(\left(\mathrm{a}_{1}, \mathrm{~b}_{3}\right), \varnothing\right),\left(\left(\mathrm{a}_{2}, \mathrm{~b}_{1}\right),\left\{\mathrm{h}_{6}\right\}\right) \\
&\left(\left(\mathrm{a}_{2}, \mathrm{~b}_{2}\right), \varnothing\right),\left(\left(\mathrm{a}_{2}, \mathrm{~b}_{3}\right), \varnothing\right) \\
&\left(\left(\mathrm{a}_{3}, \mathrm{~b}_{1}\right), \varnothing\right),\left(\left(\mathrm{a}_{3}, \mathrm{~b}_{2}\right), \varnothing\right) \\
&\left.\left(\left(\mathrm{a}_{3}, \mathrm{~b}_{3}\right), \varnothing\right)\right\} .
\end{aligned}
$$

Definition 21: If (F, A) and ( $\mathrm{G}, \mathrm{B})$ are two multiparameterized soft sets, then "(F, A) OR (G, B)" denoted by $(F, A) \vee(G, B)$ is defined by:

$$
(\mathrm{F}, \mathrm{A}) \vee(\mathrm{G}, \mathrm{B})=(\mathrm{H}, \mathrm{A} \times \mathrm{B})
$$

where $H(\alpha, \beta)=F(\alpha) \cup G(\beta) \forall(\alpha, \beta) \in A \times B$.

Example 11: Let us consider multiparameterized soft sets (F, A) and (G, B) which describes the "attractiveness of houses in the state of Selangor, Malaysia" that Mr. X and Mr. Y considering for purchase respectively over the same universe $U=\left\{h_{1}\right.$, $\left.\mathrm{h}_{2}, \mathrm{~h}_{3}, \mathrm{~h}_{4}, \mathrm{~h}_{5}, \mathrm{~h}_{6}, \mathrm{~h}_{7}, \mathrm{~h}_{8}, \mathrm{~h}_{9}, \mathrm{~h}_{10},\right\}$ such that $\mathrm{A}=\left\{\alpha_{1}=\right.$ $\left.\left\{\mathrm{e}_{1,1}\right\}, \alpha_{2}=\left\{\mathrm{e}_{1,1}, \mathrm{e}_{2,1}\right\}, \alpha_{3}=\left\{\mathrm{e}_{1,1}, \mathrm{e}_{2,1}, \mathrm{e}_{3,1}\right\}\right\} \quad B=\left\{\mathrm{e}_{1,1}\right.$, $\left.\left.e_{2,1}\right\}, b_{2}=\left\{E_{1,3}\right\}, B_{3}=\left\{E_{1,4}, E_{2,4}\right\}\right\}$. Suppose that $F\left(\alpha_{1}\right)$ $=\left\{\mathrm{h}_{2}, \mathrm{~h}_{3}, \mathrm{~h}_{6}, \mathrm{~h}_{7}, \mathrm{~h}_{10}\right\}, \mathrm{F}\left(\alpha_{2}\right)=\left\{\mathrm{H}_{6}, \mathrm{H}_{7}\right\}$ and $\mathrm{F}\left(\alpha_{3}\right)=\varnothing$ and $\mathrm{G}\left(\mathrm{b}_{1}\right)=\left\{\mathrm{h}_{2}, \mathrm{~h}_{4}, \mathrm{~h}_{6}, \mathrm{~h}_{8}\right\}, \mathrm{G}\left(\mathrm{b}_{2}\right)=\left\{\mathrm{H}_{10}\right\}$ and $\mathrm{G}\left(\mathrm{b}_{3}\right)$ $=\varnothing$, Then:

$$
\begin{aligned}
A \times B=\left\{\left(a_{1}, b_{1}\right),\left(a_{1}, b_{2}\right),\left(a_{1}, b_{3}\right),\left(a_{2}, b_{1}\right),\left(a_{2}, b_{2}\right),\right. \\
\\
\left.\left(a_{2}, b_{3}\right),\left(a_{3}, b_{1}\right),\left(a_{3}, b_{2}\right),\left(a_{3}, b_{3}\right)\right\}
\end{aligned}
$$

Hence the $(\mathrm{F}, \mathrm{A}) \vee(\mathrm{G}, \mathrm{B})$ over a common universe $\mathrm{U}$ is $(\mathrm{H}, \mathrm{A} \times \mathrm{B})$ : 


$$
\begin{aligned}
& \left\{\left(\left(\mathrm{a}_{1}, \mathrm{~b}_{1}\right),\left\{\mathrm{h}_{2}, \mathrm{~h}_{3}, \mathrm{~h}_{4}, \mathrm{~h}_{6}, \mathrm{~h}_{7}, \mathrm{~h}_{8}, \mathrm{~h}_{10}\right\}\right),\right. \\
& \left(\left(\mathrm{a}_{1}, \mathrm{~b}_{2}\right),\left\{\mathrm{h}_{2}, \mathrm{~h}_{3}, \mathrm{~h}_{6}, \mathrm{~h}_{7}, \mathrm{~h}_{10}\right\}\right), \\
& \left(\left(\mathrm{a}_{1}, \mathrm{~b}_{3}\right),\left\{\mathrm{h}_{2}, \mathrm{~h}_{3}, \mathrm{~h}_{6}, \mathrm{~h}_{7}, \mathrm{~h}_{10}\right\}\right), \\
& \left(\left(\mathrm{a}_{2}, \mathrm{~b}_{1}\right),\left\{\mathrm{h}_{2}, \mathrm{~h}_{4}, \mathrm{~h}_{6}, \mathrm{~h}_{7}, \mathrm{~h}_{8}\right\}\right), \\
& \left(\left(\mathrm{a}_{2}, \mathrm{~b}_{2}\right),\left\{\mathrm{h}_{6}, \mathrm{~h}_{7}, \mathrm{~h}_{10}\right\}\right),\left(\left(\mathrm{a}_{2}, \mathrm{~b}_{3}\right),\left\{\mathrm{h}_{6}, \mathrm{~h}_{7}\right\}\right), \\
& \left(\left(\mathrm{a}_{3}, \mathrm{~b}_{1}\right),\left\{\mathrm{h}_{2}, \mathrm{~h}_{4}, \mathrm{~h}_{6}, \mathrm{~h}_{8}\right\}\right), \\
& \left.\left(\left(\mathrm{a}_{3}, \mathrm{~b}_{2}\right),\left\{\mathrm{h}_{10}\right\}\right),\left(\left(\mathrm{a}_{3}, \mathrm{~b}_{3}\right), \varnothing\right)\right\} .
\end{aligned}
$$

Proposition 2: If (F, A) and (G, B) are two multiparameterized soft sets over $\mathrm{U}$ then:

- $((\mathrm{F}, \mathrm{A}) \wedge(\mathrm{G}, \mathrm{B}))^{\mathrm{c}}=(\mathrm{F}, \mathrm{A})^{\mathrm{c}} \vee(\mathrm{G}, \mathrm{B})^{\mathrm{c}}$

- $((\mathrm{F}, \mathrm{A}) \vee(\mathrm{G}, \mathrm{B}))^{\mathrm{c}}=(\mathrm{F}, \mathrm{A})^{\mathrm{c}} \wedge(\mathrm{G}, \mathrm{B})^{\mathrm{c}}$

Proof: See Maji et al. (2003).

Proposition 3: If $(\mathrm{F}, \mathrm{A}),(\mathrm{G}, \mathrm{B})$ and $(\mathrm{H}, \mathrm{C})$ are three multiparameterized soft sets over $\mathrm{U}$, then:

- $(\mathrm{F}, \mathrm{A}) \wedge((\mathrm{G}, \mathrm{B}) \wedge(\mathrm{H}, \mathrm{C}))=((\mathrm{F}, \mathrm{A}) \wedge(\mathrm{G}, \mathrm{B})) \wedge(\mathrm{H}, \mathrm{C})$,

- $(\mathrm{F}, \mathrm{A}) \vee((\mathrm{G}, \mathrm{B}) \vee(\mathrm{H}, \mathrm{C}))=((\mathrm{F}, \mathrm{A}) \vee(\mathrm{G}, \mathrm{B})) \vee(\mathrm{H}, \mathrm{C})$,

- $(\mathrm{F}, \mathrm{A}) \vee((\mathrm{G}, \mathrm{B}) \wedge(\mathrm{H}, \mathrm{C}))=$

$$
((\mathrm{F}, \mathrm{A}) \vee(\mathrm{G}, \mathrm{B})) \wedge((\mathrm{F}, \mathrm{A}) \vee(\mathrm{H}, \mathrm{C})) \text {, }
$$

- $(\mathrm{F}, \mathrm{A}) \wedge((\mathrm{G}, \mathrm{B}) \vee(\mathrm{H}, \mathrm{C}))=$

$((\mathrm{F}, \mathrm{A}) \wedge(\mathrm{G}, \mathrm{B})) \vee((\mathrm{F}, \mathrm{A}) \wedge(\mathrm{H}, \mathrm{C}))$.

Proof: Straightforward from definitions 20 and 21.

\section{ACKNOWLEDGEMENT}

The authors would like to acknowledge the financial support received from University Kebangsaan Malaysia under the research grant UKM-ST-06FRGS0104-2009.

\section{REFERENCES}

Ali, M.I., F. Feng, X. Liu, W.K. Min and M. Shabir, 2009. On some new operations in soft set theory. Comput. Math. Appli., 57: 1547-1553. DOI: 10.1016/j.camwa.2008.11.009

Chen, D., E.C.C. Tsang, D.S. Yeung and X. Wang, 2005. The parameterization reduction of soft sets and its application. Comput. Math. Appli., 49: 757763. DOI: 10.1016/j.camwa.2004.10.036

Maji, P.K., A.R. Roy and R. Biswas, 2001. Fuzzy soft sets. J. Fuzzy Math., 9: 589-602.

Maji, P.K., A.R. Roy and R. Biswas, 2002. An application of soft sets in a decision making problem. Comput. Math. Appli., 44: 1077-1083. DOI: 10.1016/S0898-1221(02)00216-X

Maji, P.K., R. Biswas and A.R. Roy, 2003. Soft set theory. Comput. Math. Appli., 45: 555-562. DOI: 10.1016/S0898-1221(03)00016-6

Molodtsov, D., 1999. Soft set theory-first results. Comput. Math. Appli., 37: 19-31. DOI: 10.1016/S0898-1221(99)00056-5

Roy, A.R. and P.K. Maji, 2007. A fuzzy soft set theoretic approach to decision making problems. J. Comput. Applied Math., 203: 412-418. DOI: 10.1016/j.cam.2006.04.008 